\title{
Medication Prescribing Quality in Australian Primary Care Patients with Chronic Kidney Disease
}

\author{
Woldesellassie M. Bezabhe* ${ }^{*}$, Alex Kitsos, Timothy Saunder, Gregory M. Peterson $\mathbb{D}$, \\ Luke R. Bereznicki, Barbara C. Wimmer, Matthew Jose and Jan Radford $\mathbb{D}$
}

School of Pharmacy and Pharmacology, University of Tasmania, Private Bage 26, Hobart, Tasmania 7001, Australia; alex.kitsos@utas.edu.au (A.K.); tim.saunder@utas.edu.au (T.S.); g.peterson@utas.edu.au (G.M.P.); luke.bereznicki@utas.edu.au (L.R.B.); barbara.wimmer@utas.edu.au (B.C.W.); Matthew.Jose@utas.edu.au (M.J.); J.Radford@utas.edu.au (J.R.)

* Correspondence: woldesellassie.bezabhe@utas.edu.au

Received: 3 February 2020; Accepted: 8 March 2020; Published: 13 March 2020

\begin{abstract}
Background: Australian patients with chronic kidney disease (CKD) are routinely managed in general practices with multiple medications. However, no nationally representative study has evaluated the quality of prescribing in these patients. The objective of this study was to examine the quality of prescribing in patients with CKD using nationally representative primary care data obtained from the NPS MedicineWise's dataset, MedicineInsight. Methods: A cross-sectional analysis of general practice data for patients aged 18 years or older with CKD was performed from 1 February 2016 to 1 June 2016. The study examined the proportion of patients with CKD who met a set of 16 published indicators in two categories: (1) potentially appropriate prescribing of antihypertensives, renin-angiotensin system (RAS) inhibitors, phosphate binders, and statins; and (2) potentially inappropriate prescribing of nephrotoxic medications, such as non-steroidal anti-inflammatory drugs (NSAIDs), at least two RAS inhibitors, triple therapy (an NSAID, a RAS inhibitor and a diuretic), high-dose digoxin, and metformin. The proportion of patients meeting each quality indicator was stratified using clinical and demographic characteristics. Results: A total of 44,259 patients $(24,165$ (54.6\%) female; 25,562 (57.8\%) estimated glomerular filtration (eGFR) $45-59 \mathrm{~mL} / 1.73 \mathrm{~m}^{2}$ ) with CKD stages 3-5 were included. Nearly one-third of patients had diabetes and were more likely to have their blood pressure and albumin-to-creatinine ratio monitored than those without diabetes. Potentially appropriate prescribing of antihypertensives was achieved in $79.9 \%$ of hypertensive patients with CKD stages 4-5. The prescribing indicators for RAS inhibitors in patients with microalbuminuria and diabetes and in patients with macroalbuminuria were achieved in $69.9 \%$ and $62.3 \%$ of patients, respectively. Only $40.8 \%$ of patients with CKD and aged between 50 and 65 years were prescribed statin therapy. The prescribing of a RAS inhibitor plus a diuretic was less commonly achieved, with the indicator met in $20.6 \%$ for patients with microalbuminuria and diabetes and $20.4 \%$ for patients with macroalbuminuria. Potentially inappropriate prescribing of NSAIDs, metformin, and at least two RAS inhibitors were apparent in $14.3 \%, 14.1 \%$, and $7.6 \%$, respectively. Potentially inappropriate prescribing tended to be more likely in patients aged $\geq 65$ years, living in regional or remote areas, or with socio-economic indexes for areas (SEIFA) score $\leq 3$. Conclusions: We identified areas for possible improvement in the prescribing of RAS inhibitors and statins, as well as deprescribing of NSAIDs and metformin in Australian general practice patients with CKD.
\end{abstract}

Keywords: chronic kidney disease; drug therapy; quality indicators; appropriate prescribing; general practice; quality use of medicine; primary care 


\section{Background}

In 2015, an estimated 1.7 million Australian adults aged 18 years or older had indicators of chronic kidney disease (CKD), and of these, 604,000 had CKD stages 3-5 [1]. Diabetes and hypertension caused up to two-thirds of CKD cases. Approximately one in three and three in four Australian general practice patients with CKD had recorded diagnoses of diabetes and hypertension, respectively [2]. Progressive kidney damage with hypertension and diabetes leads to end-stage kidney disease (ESKD) [3]. In 2016 alone, 2800 new cases of ESKD were reported in Australia [4]. Patients with ESKD require expensive replacement therapy, and their treatment costs the Australian economy 1 billion per year [1].

Prevention of CKD progression is cost-effective and is most successful within primary care [3]. In Australia, the majority of patients with CKD stages 3-5 receive treatment from general practices [2]. Prevention of CKD progression can be achieved by treatment of modifiable risk factors and avoidance of nephrotoxic medication [3]. Kidney Health Australia's 'CKD management in general practice' guideline recommends, depending on the stage of $C K D$, adequate treatment of hypertension, dyslipidaemia, and albuminuria [3]. It recommends controlling blood pressure at $\leq 140 / 90 \mathrm{~mm} \mathrm{Hg}$ in patients with CKD alone and $\leq 130 / 80 \mathrm{~mm} \mathrm{Hg}$ in those comorbid with albuminuria or diabetes [3].

Angiotensin-converting enzyme inhibitors (ACEIs) or angiotensin receptor blockers (ARBs) are first-line antihypertensive agents in CKD patients with albuminuria or diabetes [3]. These classes of drugs not only lower blood pressure but also decrease the progression of albuminuria [3]. Statin therapy is recommended in patients with CKD and aged 50 years or older as it reduces cardiovascular risk and progression of CKD [5,6]. Australian guidelines also recommend avoiding the use of medications that can potentially damage kidney function or readily accumulate and cause toxicity [6]. These medications include non-steroidal anti-inflammatory drugs (NSAID), metformin, and a high dose of digoxin [3].

There is limited research investigating the quality of CKD care in Australian patients [7-10]. The available studies have focused on specific classes of medications [8,9] and specific regions $[7,9]$ or single centres [10] that may not be generalisable to the broader CKD population in Australia. There remains a need to assess prescribing quality with validated indicators in patients with CKD. Smits et al. developed a set of 16 prescribing quality indicators (PQIs) [11], which were developed according to international guidelines recommendations and are relevant to evaluate the quality of CKD care in a primary care setting $[12,13]$. We aimed to evaluate the quality of Australian prescribing with these indicators in patients with CKD using a large and nationally representative general practice dataset.

\section{Methods}

We analysed retrospective data obtained from the NPS MedicineWise MedicineInsight dataset. The data were de-identified and extracted from the electronic health records (EHRs) of general practices and included demographics, encounters, diagnoses, prescriptions, observations, and pathology tests. NPS MedicineWise MedicineInsight is the largest geographically representative primary care dataset in Australia. As of October 2018, NPS MedicineWise MedicineInsight had recruited 671 general practices across Australia. A total of 2,974,031 included patients had at least three clinical encounters in the previous two years. Details about this dataset can be found elsewhere [2,14-16]. We used MedicineInsight data collected from 329 general practices between 1 January 2013 and 1 June 2016.

In this study, we included patients with evidence of CKD based on having two renal function tests that were performed at least three months apart with: (1) estimated glomerular filtration rate (eGFR) values $<60 \mathrm{~mL} / \mathrm{min} / 1.73 \mathrm{~m}^{2}$ or (2) albumin-to-creatinine ratio (ACR) values $\geq 3.5 \mathrm{mg} / \mathrm{mmol}$ for females or $\geq 2.5 \mathrm{mg} / \mathrm{mmol}$ for males. The renal function tests were performed between 1 January 2013 and 1 June 2015. The CKD epidemiology collaboration equation (CKD-EPI) was used to calculate eGFR [17]. This definition of CKD is congruent with that recommended for the diagnosis of CKD in Australian general practice [3]. Regular patients (defined by the Royal Australian College of General Practitioners as those with three or more encounters in the previous two years) [16] were included, if at the time of data extraction (July 2016) they were aged at least 18 years. Patients were excluded 
if they did not have at least one follow-up general practitioner (GP) visit between 2 June 2015 and 1 June 2016, in addition to patients who died during that period.

Variables such as age, gender, socio-economic status (based on the Index of Relative Socio-economic Advantage and Disadvantage, one of the socio-economic indexes for areas (SEIFA)) [18], rurality, continuity of care $(\mathrm{CoC})$, documentation of a diagnosis of CKD, and serum electrolyte levels (e.g., calcium, and phosphate) were examined. SEIFA quintile was an index developed by the Australian Bureau of Statistics (ABS) and ranks areas in Australia from 1 (most disadvantaged area) to 5 (most advantaged area) [18]. Rurality was assigned according to the postcode of the patient's residence and classified as major cities, regional, remote, and very remote Australia [19]. SEIFA quintile was categorised into SEIFA $\leq 3$ vs. SEIFA $>3$ and rurality to major cities vs. regional and remote Australia. CoC was calculated for each included patient after the time of laboratory evidence of CKD, over the remainder of the data collection period, using the Herfindahl-Hirschman Index, which has been shown to be highly correlated with other common measures of $\mathrm{CoC}$ [20]. Its value ranged from 0 to 1 and cut off points for low and high $\mathrm{CoC}$ were $<0.75$ and $>=0.75$, respectively. Low $\mathrm{CoC}$ in general practice, measured with this index, has also been associated with a higher risk of mortality [21,22].

Documentation of a diagnosis of CKD was extracted from condition codes. Baseline comorbidities, including myocardial infarction, hypertension, and diabetes were examined. The comorbidities were based on 'condition flags' provided by MedicineInsight. The prescribed medications that were examined included: diuretics (anatomical therapeutic chemical (ATC) code: C03), beta-blockers (C07), calcium channel blockers (C08), ACEIs (C09A), ARBs (C09C), other agents acting on renin-angiotensin system (RAS) (C09), other antihypertensives (C02), statins (C10AA or combinations as in C10BA and C10BX), phosphate binders (A12AA04, A12AA12, V03AE, and A02AB01), erythropoiesis-stimulating agents (ESAs) (B03XA), non-steroidal anti-inflammatory drugs (NSAIDs) (M01A, M01BA and B01AC), metformin (A10BA02 or in combination as A10BD), and digoxin (C01AA05).

The recorded data included prescriptions and laboratory tests during the last four months of follow-up (between 1 February 2016 and 1 June 2016). The 16 PQIs, developed and validated by Smits et al. [11] in the Netherlands, were used to evaluate this data. They were categorised into two domains: appropriate and inappropriate PQIs. Detailed definitions for all indicators are shown in Table 4. The appropriate prescribing domain includes the first nine indicators that evaluate prescribing of all antihypertensive agents in patients with hypertension, RAS inhibitors, or diuretics in patients with proteinuria or diabetes, statins, and phosphate binders. The inappropriate prescribing domain contains the remaining seven indicators that assess the prescribing of ESA in patients with CKD and haemoglobin $\geq 7.5 \mathrm{~g} / \mathrm{dL}$, use of NSAIDs and metformin in patients with eGFR $<30 \mathrm{~mL} / \mathrm{min} / 1.73 \mathrm{~m}^{2}$, high-dose digoxin in patients with eGFR $<50 \mathrm{~mL} / \mathrm{min} / 1.73 \mathrm{~m}^{2}$, simultaneous use of at least two RAS inhibitors, and triple therapy with an NSAID, RAS inhibitor, and diuretic. The use of phosphate binders and ESAs was not captured in our dataset as nephrologists in Australia typically prescribe them.

Simultaneous prescribing of RAS blockers was defined as at least two of the ATC codes C09A, C09B, C09C, C09D, C09X, or combination (as in C10BX) within the last four months of the follow-up (between 1 February 2016 and 1 June 2016). Simultaneous use of NSAID, RAS blocker, and diuretic was defined as at least one prescription for each of the three classes of medications during the follow-up period. It was acknowledged that we were not capable of capturing the use of over-the-counter NSAIDs. We could also not capture whether NSAIDs were prescribed as a regular medication or for 'as needed' use.

\subsection{Statistical Analysis}

Patient demographics and clinical characteristics were compared between patients with and without diabetes and are presented as numbers and proportions. The proportion of patients who met PQIs criteria are shown as percentages with $95 \%$ confidence intervals (CIs). We stratified the proportion of patients meeting each indicator by gender, age, SEIFA, rurality, CKD diagnosis documentation, and $\mathrm{CoC}$. Chi-square tests were used to determine differences in the quality of prescribing with patient 
characteristics. All data analyses and management were conducted using the statistical and graphical computing language of $\mathrm{R}$ [23]. A two-sided $p$ value of less than 0.05 was considered to indicate statistical significance.

\subsection{Ethics Approval and Consent to Participate}

Ethics approval was obtained from the Tasmanian Health and Medical Human Research Ethics Committee (H0015651). De-identified data obtained from the MedicineInsight for this study did not have any patient-specific information, such as date of birth, age and postcode and individual patient consent was waived for our ethics application. Patients were informed about the programme through promotional material that was displayed with the waiting room of all participating practices. Patients choice to opt-out from the programme was respected, and robust and effective security controls safeguarded their safety.

\section{Results}

\subsection{Baseline Charactersitics}

The cohort was composed of 44,259 patients with evidence of CKD. Of these, 24,165 (54.6\%) were females, and $70 \%$ were aged 70 years or older. Most patients (57.8\%) had eGFR values between 45 and $59 \mathrm{~mL} / \mathrm{min} / 1.73 \mathrm{~m}^{2}$. Only a quarter of patients with evidence of CKD had documentation of the diagnosis, and documentation was less likely with increasing age (e.g., 51.3\% for patients aged $30-39$ years with evidence of CKD vs. $23.9 \%$ in those aged $\geq 80$ years; $p<0.001)$. The sociodemographic and clinical characteristics of the study participants, including medications prescribed and monitoring performed, are shown in Tables 1-3.

A total of 13,263 patients (30\%) had diabetes. Of these, 11,608 (87.5\%) and $6608(87.5 \%)$ had hypertension and a history of myocardial infarction, respectively (Table 2). Of 39,716 (89.7\%) patients who had a recorded blood pressure measurement, 13,338 (33.6\%) had uncontrolled blood pressure ( $>140 / 90 \mathrm{~mm} \mathrm{Hg}$ ). The proportion of patients with uncontrolled blood pressure was slightly higher in patients with diabetes $(34.4 \%$ vs. $33.2 \%, p=0.03)$ than in those without diabetes. Antihypertensive medication prescribing was significantly higher in CKD patients with diabetes compared with those without diabetes $(82.1 \%$ vs. $70.6 \%, p<0.001)$. Compared with CKD patients without diabetes, CKD patients with diabetes were more likely to be prescribed an ACEI/ARB $(64.1 \%$ vs. $51.5 \%, p<0.001)$. Over $60 \%$ of CKD patients with diabetes were prescribed a statin compared with less than $40 \%$ without diabetes $(p<0.001)$ (Table 3$)$.

Only a few patients had recorded treatment with phosphate binders, ESAs, and vitamin D. Therefore, the three PQIs: seven, eight, and nine that assess appropriate prescribing of phosphate binders and the two PQIs: 11 and 12 that evaluate inappropriate prescribing of vitamin D and ESAs were not operational. These five indicators were excluded from further analyses. 
Table 1. Baseline sociodemographic characteristics of patients with chronic kidney disease (CKD) overall and by diabetes status.

\begin{tabular}{|c|c|c|c|c|}
\hline & \multirow{2}{*}{$\begin{array}{l}\text { Overall, } n= \\
44,259 \mathrm{n}(\%)\end{array}$} & \multicolumn{2}{|c|}{ Diabetes } & \multirow[b]{2}{*}{$p$ Value } \\
\hline & & $\begin{array}{c}\text { No } n=30,996 \\
n(\%)\end{array}$ & $\begin{array}{c}\text { Yes } n=13,263 \\
n(\%)\end{array}$ & \\
\hline Age groups (years) & & & & $<0.836$ \\
\hline$<65$ & 4373 (9.9) & 3069 (9.9) & $1304(9.8)$ & \\
\hline$\geq 65$ & $39,886(90.1)$ & $27,927(90.1)$ & $11,959(90.2)$ & \\
\hline Female & $24,165(54.6)$ & $17,620(56.8)$ & $6545(49.3)$ & $<0.001$ \\
\hline Indigenous Status & & & & $<0.001$ \\
\hline Indigenous & $436(1.0)$ & $212(0.7)$ & $224(1.7)$ & \\
\hline Non-Indigenous & $33,067(74.7)$ & $23,020(74.3)$ & $10,047(75.8)$ & \\
\hline Missing & $10,756(24.3)$ & $7764(25.0)$ & $2992(22.6)$ & \\
\hline \multicolumn{5}{|l|}{ SEIFA quintile * } \\
\hline$\leq 3$ & $12,254(27.8)$ & $8302(26.9)$ & $3952(30.0)$ & $<0.001$ \\
\hline$>3$ & $31,754(72.2)$ & $22,559(73.1)$ & $9225(70.0)$ & \\
\hline Missing & $251(0.6)$ & $165(0.5)$ & $86(0.6)$ & \\
\hline Rurality * & & & & $<0.001$ \\
\hline Major Cities of Australia & $26,617(60.4)$ & $18,468(59.9)$ & $8149(61.8)$ & \\
\hline Regional and Remote Australia & $17,420(39.6)$ & $12,385(40.1)$ & $5035(38.2)$ & \\
\hline Missing & $222(0.5)$ & $143(0.5)$ & $79(0.6)$ & \\
\hline GP Continuity of Care & & & & $<0.001$ \\
\hline Low $(<0.75)$ & $17,421(39.4)$ & $11,917(38.5)$ & $5504(41.5)$ & \\
\hline $\operatorname{High}(\geq 0.75)$ & $26,833(60.6)$ & $19,075(61.5)$ & $7758(58.5)$ & \\
\hline Missing & $5(0.0)$ & $1(0.0)$ & $4(0.0)$ & \\
\hline Documentation of CKD & $11,618(26.3)$ & $7722(24.9)$ & $3896(29.4)$ & $<0.001$ \\
\hline
\end{tabular}

SEIFA, socio-economic indexes for areas; GP, general practitioner. ${ }^{*}$ Excludes patients without a recorded postcode in the electronic health record.

Table 2. Comorbidities of patients with CKD overall and by diabetes status.

\begin{tabular}{|c|c|c|c|c|}
\hline & \multirow[b]{2}{*}{$\begin{array}{l}\text { Overall, } n= \\
44,259 \mathrm{n}(\%)\end{array}$} & \multicolumn{2}{|c|}{ Diabetes } & \multirow[b]{2}{*}{$p$ Value } \\
\hline & & $\begin{array}{c}\text { No } n=30,996 \\
n(\%)\end{array}$ & $\begin{array}{c}\text { Yes } n=13,263 \\
n(\%)\end{array}$ & \\
\hline CKD Stage & & & & $<0.001$ \\
\hline Stage 3a $\left(45-59 \mathrm{~mL} / \mathrm{min} / 1.73 \mathrm{~m}^{2}\right)$ & $25,562(57.8)$ & $18,724(60.4)$ & 6838 (51.6) & \\
\hline Stage $3 \mathrm{~b}\left(30-44 \mathrm{~mL} / \mathrm{min} / 1.73 \mathrm{~m}^{2}\right)$ & $13,551(30.6)$ & $9093(29.3)$ & 4458 (33.6) & \\
\hline Stage $4\left(15-29 \mathrm{~mL} / \mathrm{min} / 1.73 \mathrm{~m}^{2}\right)$ & $4186(9.5)$ & $2573(8.3)$ & $1613(12.2)$ & \\
\hline Stage $5\left(<15 \mathrm{~mL} / \mathrm{min} / 1.73 \mathrm{~m}^{2}\right)$ & $960(2.2)$ & $606(2.0)$ & $354(2.7)$ & \\
\hline $\mathrm{ACR}(\mathrm{mg} / \mathrm{mmol})$ & & & & 0.023 \\
\hline $\begin{array}{c}\text { Normal } \\
<2.5 \text { (male) } \\
<3.5 \text { (female) }\end{array}$ & $7877(17.8)$ & $3838(12.4)$ & 4039 (30.5) & \\
\hline $\begin{array}{c}\text { Microalbuminuria } \\
2.5-25 \text { (male) } \\
3.5-35 \text { (female) }\end{array}$ & 4707 (10.6) & $1719(5.6)$ & $2978(22.5)$ & \\
\hline $\begin{array}{c}\text { Macroalbuminuria } \\
>25 \text { (male) } \\
>35 \text { (female) }\end{array}$ & $2427(5.5)$ & $897(2.9)$ & $1530(11.5)$ & \\
\hline Missing & $29,248(66.1)$ & $24,532(79.1)$ & $4716(35.6)$ & \\
\hline Indigenous Status & & & & $<0.001$ \\
\hline $\begin{array}{c}\text { Indigenous } \\
\text { Non-Indigenous } \\
\text { Missing } \\
\text { Comorbidities }\end{array}$ & $\begin{array}{c}436(1.0) \\
33,067(74.7) \\
10,756(24.3)\end{array}$ & $\begin{array}{c}212(0.7) \\
23,020(74.3) \\
7764(25.0)\end{array}$ & $\begin{array}{c}224(1.7) \\
10,047(75.8) \\
2992(22.6)\end{array}$ & \\
\hline
\end{tabular}


Table 2. Cont.

\begin{tabular}{|c|c|c|c|c|}
\hline & \multirow{2}{*}{$\begin{array}{l}\text { Overall, } n= \\
44,259 \text { n (\%) }\end{array}$} & \multicolumn{2}{|c|}{ Diabetes } & \multirow[b]{2}{*}{$p$ Value } \\
\hline & & $\begin{array}{c}\text { No } n=30,996 \\
n(\%)\end{array}$ & $\begin{array}{c}\text { Yes } n=13,263 \\
n(\%)\end{array}$ & \\
\hline Hypertension & $35,386(80.0)$ & $23,778(76.7)$ & $11,608(87.5)$ & $<0.001$ \\
\hline Myocardial infarction & $17,945(40.5)$ & $11,688(37.7)$ & $6257(47.2)$ & $<0.001$ \\
\hline Atrial fibrillation & 7038 (15.9) & $4893(15.8)$ & $2145(16.2)$ & 0.315 \\
\hline Anxiety & $5658(12.8)$ & $4124(13.3)$ & $1534(11.6)$ & $<0.001$ \\
\hline Bipolar disorder & $505(1.1)$ & $365(1.2)$ & $140(1.1)$ & 0.290 \\
\hline Schizophrenia & $363(0.8)$ & $227(0.7)$ & $136(1.0)$ & 0.002 \\
\hline
\end{tabular}

$\mathrm{ACR}$, albumin-to-creatinine ratio; $\mathrm{CKD}$, chronic kidney disease.

Table 3. Proportion of patients with CKD receiving monitoring and medications by diabetes status.

\begin{tabular}{|c|c|c|c|c|}
\hline & \multirow[b]{2}{*}{$\begin{array}{c}\text { Total } n= \\
44,259 \mathrm{n}(\%)\end{array}$} & \multicolumn{2}{|c|}{ Diabetes } & \multirow[b]{2}{*}{$p$ Value } \\
\hline & & $\begin{array}{c}\text { No } n=30,996 \\
n(\%)\end{array}$ & $\begin{array}{c}\text { Yes } n=13,263 \\
n(\%)\end{array}$ & \\
\hline \multicolumn{5}{|l|}{ Blood Pressure } \\
\hline Patients with BP Recorded & $39,716(89.7)$ & $27,411(88.4)$ & $12,305(92.8)$ & $<0.001$ \\
\hline Low Diastolic BP (<70 mmHg) & $13,602(34.2)$ & 8935 (32.6) & $4667(37.9)$ & $<0.001$ \\
\hline High Systolic BP (>140 mmHg) & $13,338(33.6)$ & 9108 (33.2) & $4230(34.4)$ & 0.033 \\
\hline \multicolumn{5}{|l|}{ Pathology } \\
\hline $\begin{array}{l}\text { Patients with phosphate test } \\
\text { recorded }\end{array}$ & $23,133(52.3)$ & $16,060(51.8)$ & $7073(53.3)$ & 0.004 \\
\hline $\begin{array}{c}\text { Elevated phosphate level }(>1.49 \\
\mathrm{mmol} / \mathrm{L})\end{array}$ & $1322(5.7)$ & $872(5.4)$ & $450(6.4)$ & 0.005 \\
\hline $\begin{array}{l}\text { Patients with calcium test } \\
\text { recorded }\end{array}$ & $22,818(51.6)$ & $16,096(51.9)$ & $6722(50.7)$ & 0.017 \\
\hline $\begin{array}{c}\text { Elevated calcium level (>2.54 } \\
\mathrm{mmol} / \mathrm{L})\end{array}$ & $1343(5.9)$ & $893(5.5)$ & $450(6.7)$ & $<0.001$ \\
\hline Low calcium level $(<2.10 \mathrm{mmol} / \mathrm{L})$ & $589(2.6)$ & $409(2.5)$ & $180(2.7)$ & 0.584 \\
\hline Patients with $\mathrm{Hb}$ test recorded & $40,601(91.7)$ & $28,723(92.7)$ & $11,878(89.6)$ & $<0.001$ \\
\hline Low $\mathrm{Hb}$ level $(<7.5 \mathrm{mmol} / \mathrm{L})$ & $14,125(34.8)$ & $9252(32.2)$ & $4873(41.0)$ & $<0.001$ \\
\hline \multicolumn{5}{|l|}{ Medication } \\
\hline \multicolumn{5}{|l|}{ Antihypertensives } \\
\hline At least one antihypertensives ${ }^{\neq}$ & $32,782(74.1)$ & $21,893(70.6)$ & $10,889(82.1)$ & $<0.001$ \\
\hline Diuretic & $9539(21.6)$ & $5956(19.2)$ & $3583(27.0)$ & $<0.001$ \\
\hline Beta Blocker & $10,763(24.3)$ & $6862(22.1)$ & $3901(29.4)$ & $<0.001$ \\
\hline Calcium Channel Blocker & $9551(21.6)$ & $6232(20.1)$ & $3319(25.0)$ & $<0.001$ \\
\hline ACEI or ARB & $24,485(55.3)$ & $15,978(51.5)$ & $8507(64.1)$ & $<0.001$ \\
\hline Multiple ACEI or ARB & $1859(4.2)$ & $1066(3.4)$ & $793(6.0)$ & $<0.001$ \\
\hline Statin & $20,411(46.1)$ & $12,370(39.9)$ & $8041(60.6)$ & $<0.001$ \\
\hline All phosphate binders & $244(0.6)$ & $155(0.5)$ & $89(0.7)$ & 0.031 \\
\hline $\begin{array}{l}\text { Non-calcium-containing } \\
\text { phosphate binders }\end{array}$ & $67(0.2)$ & $41(0.1)$ & $26(0.2)$ & 0.148 \\
\hline $\begin{array}{l}\text { Calcium-containing phosphate } \\
\text { binders }\end{array}$ & $182(0.4)$ & $119(0.4)$ & $63(0.5)$ & 0.197 \\
\hline
\end{tabular}


Table 3. Cont.

\begin{tabular}{|c|c|c|c|c|}
\hline & \multirow[b]{2}{*}{$\begin{array}{c}\text { Total } n= \\
44,259 \text { n (\%) }\end{array}$} & \multicolumn{2}{|c|}{ Diabetes } & \multirow[b]{2}{*}{$p$ Value } \\
\hline & & $\begin{array}{c}\text { No } n=30,996 \\
n(\%)\end{array}$ & $\begin{array}{c}\text { Yes } n=13,263 \\
n(\%)\end{array}$ & \\
\hline Vitamin D & $1444(3.3)$ & 939 (3.0) & 505 (3.8) & $<0.001$ \\
\hline ESAs & $42(0.1)$ & $24(0.1)$ & $18(0.1)$ & 0.098 \\
\hline NSAIDs & $7426(16.8)$ & 4862 (15.7) & 2564 (19.3) & $<0.001$ \\
\hline Metformin & 5189 (11.7) & $59 *(0.2)$ & $5130(38.7)$ & $<0.001$ \\
\hline Digoxin & $1516(3.4)$ & $976(3.1)$ & $540(4.1)$ & $<0.001$ \\
\hline
\end{tabular}

$\mathrm{BP}$, blood pressure; $\mathrm{Hb}$, haemoglobin; $\mathrm{ACEI}$, angiotensin-converting enzyme inhibitor; $\mathrm{ARB}$, angiotensin receptor blocker; ESAs, erythropoiesis-stimulating agents; NSAIDs, non-steroidal anti-inflammatory drugs. ${ }^{\neq}$Includes all antihypertensives with anatomical therapeutic chemical (ATC) code $\mathrm{C} 02, \mathrm{C} 03, \mathrm{C} 07, \mathrm{C} 08, \mathrm{C} 09$, or combinations (as in C10BX). * Patients with a prescription for metformin who did not have a recorded diagnosis of type 2 diabetes.

\subsection{Appropriate Prescribing}

Among patients with CKD stages $4-5$ and hypertension, $79.9 \%$ overall and $83.5 \%$ of those aged $\geq 65$ years were prescribed antihypertensive agents. The proportion of patients with microalbuminuria (ACR $2.5-25 \mathrm{mg} / \mathrm{mmol}$ for males, 3.5-35 mg/mmol for females) and diabetes who were prescribed an ACEI/ARB or an ACEI/ARB plus a diuretic were $69.9 \%$ and $20.6 \%$, respectively. Overall, the prescribing of ACEI/ARB in patients with macroalbuminuria (ACR $>25 \mathrm{mg} / \mathrm{mmol}$ for males, $>35 \mathrm{mg} / \mathrm{mmol}$ for females) was $62.3 \%$. This was significantly higher in those patients aged $\geq 65$ years than those $<65$ years $(65.4 \%$ vs. $56.1 \%, p<0.001)$ and in those without documented CKD diagnosis $(64.5 \%$ vs. $60.0 \%$, $p=0.046)$ than those documented. The proportion of patients with macroalbuminuria who were prescribed an ACEI/ARB plus a diuretic was $20.4 \%$, overall, and was significantly higher in those aged $\geq 65$ years $(22 \%$ vs. $16.7 \%, p=0.021)$ than those $<65$ years (Table 4 and Supplementary Figure S1).

We examined the prescribing of statins in CKD patients with diabetes and in those aged between 50 and 65 years, as guidelines recommend statin use in both of these groups [12]. The proportion of patients who were prescribed a statin was $39.9 \%$ in patients without diabetes and $60.6 \%$ in patients with diabetes. The percentage of statin prescribing was $40.8 \%$ in patients with CKD aged between 50 and 65 years. Prescribing of statins in this age group was more common in patients with a SEIFA score $\leq 3$ than $>3(45.3 \%$ vs. $38.9 \%, p<0.001)$ and in patients with a documented CKD diagnosis $(45.1 \%$ vs. $38.9 \%, p<0.001$ ) (Table 4 and Supplementary Figure S1).

\subsection{Potentially Inappropriate Prescribing}

The percentage of patients with potentially inappropriate prescribing of an NSAID in combination with a RAS blocker and a diuretic (triple therapy) was $2.6 \%$, overall. It was higher in those whose CKD diagnosis was documented ( $3.0 \%$ vs. $2.5 \%, p=0.002)$ than not documented, and in those aged $\geq 65$ years $(2.7 \%$ vs. $2.0 \%, p=0.004)$ than aged $<65$ years. It was slightly higher in patients with SEIFA score $\leq 3$ than $>3(3.2 \%$ vs. $2.4 \%, p<0.001)$ and in CKD patients living in regional and remote areas than in patients living in major cities $(2.8 \%$ vs. $2.5 \%$; $p=0.032)$ (Table 4 and Supplementary Figure S2). Among patients with eGFR $<30 \mathrm{~mL} / \mathrm{min} / 1.73 \mathrm{~m}^{2}$, the proportion prescribed an NSAID was $14.3 \%$ overall and was higher in patients aged $\geq 65$ years $(15.1$ vs. $9.4 \%, p<0.001)$ than those aged $<65$ years and in patients with SEIFA score $\leq 3(15.9 \%$ vs. $13.6 \%, p=0.033)$ than those with SEIFA score $>3$.

Of those patients with CKD stages 3-5 and prescribed a RAS blocker, $7.6 \%$ were prescribed at least two RAS blockers simultaneously. This was more likely in patients with SEIFA score $\leq 3$ than $>3$ $(8.3 \%$ vs. $7.3 \% ; p=0.005)$ and in patients living in major cities than those living in regional and remote areas $(8.0 \%$ vs. $7.0 \% ; p=0.002)$ (Table 4 and Supplementary Figure S2).

There were 5130 patients with diabetes who were prescribed metformin. Of 1967 patients with a diagnosis of diabetes and with an eGFR $<30 \mathrm{~mL} / \mathrm{min} / 1.73 \mathrm{~m}^{2}, 278(14.1 \%)$ were potentially inappropriately prescribed metformin. This was slightly greater in patients living in regional and 
remote Australia (16.8\%) than those living in major cities $(12.3 \% ; p=0.005)$ and in patients whose CKD diagnosis was not documented $(16.3 \%$ vs. $12.5 \% ; p=0.018)$ (Table 4 , Supplementary Figure S2).

In patients with an eGFR $<50 \mathrm{~mL} / \mathrm{min} / 1.73 \mathrm{~m}^{2}$, the proportion prescribed high-dose digoxin $(0.125 \mathrm{mg} /$ day) was $3.8 \%$. This was higher in females $(4.1 \%$ vs. $3.3 \%, p<0.001)$, in those aged $\geq 65$ years $(4.0 \%$ vs. $1.1 \%, p<0.001)$ than aged $<65$ years, and in those living in regional and remote areas $(4.2 \%$ vs. $3.5 \%, p=0.002)$ than those living in major cities (Table 4 and Supplementary Figure S2). 
Table 4. Number and proportion of patients meeting prescribing quality indicators by rurality, socio-economic indexes for areas (SEIFA) and CKD documentation [4].

\begin{tabular}{|c|c|c|c|c|c|c|}
\hline \multicolumn{3}{|c|}{ Quality Indicator } & Numerator & Denominator & Percentage & $p$ Value \\
\hline \multicolumn{7}{|c|}{ Treatment of Hypertension } \\
\hline \multirow{15}{*}{$\begin{array}{l}\text { 1. Percentage of } \\
\text { patients aged } 18 \text { to } 80 \\
\text { years with CKD } \\
\text { stages } 4-5 \text { and } \\
\text { hypertension who are } \\
\text { prescribed } \\
\text { antihypertensives } \\
\text { unless undesirable } \\
\text { because of low } \\
\text { diastolic blood } \\
\text { pressure }\end{array}$} & Overall * & & 1029 & 1288 & 79.9 & \\
\hline & Rurality & $\begin{array}{l}\text { Major cities of } \\
\text { Australia }\end{array}$ & 532 & 672 & 79.2 & 0.565 \\
\hline & \multirow{3}{*}{ SEIFA quintile } & $\begin{array}{c}\text { Regional and Remote } \\
\text { Australia }\end{array}$ & 490 & 609 & 80.5 & \\
\hline & & $\leq 3$ & 345 & 433 & 79.7 & 0.947 \\
\hline & & $>3$ & 677 & 848 & 79.8 & \\
\hline & \multirow{2}{*}{$\mathrm{CoC}$} & High & 375 & 459 & 81.7 & 0.228 \\
\hline & & Low & 654 & 829 & 78.9 & \\
\hline & \multirow{2}{*}{ CKD documented } & No & 380 & 485 & 78.4 & 0.284 \\
\hline & & Yes & 649 & 803 & 80.8 & \\
\hline & \multirow{2}{*}{ Systolic BP } & $>140 \mathrm{mmHg}$ & 455 & 573 & 79.4 & 0.541 \\
\hline & & $\leq 140 \mathrm{mmHg}$ & 588 & 728 & 80.8 & \\
\hline & \multirow{2}{*}{ Age } & $<65$ years & 318 & 437 & 72.8 & $<0.001$ \\
\hline & & $\geq 65$ years & 711 & 851 & 83.5 & \\
\hline & \multirow{2}{*}{ Sex } & Female & 450 & 561 & 80.2 & 0.800 \\
\hline & & Male & 579 & 727 & 79.6 & \\
\hline \multirow{15}{*}{$\begin{array}{l}\text { 2. Percentage of } \\
\text { patients aged } 18 \text { to } \\
80 \text { years with CKD } \\
\text { stages } 3-5 \text { and } \\
\text { macroalbuminuria } \\
\text { treated with multiple } \\
\text { antihypertensives } \\
\text { who are prescribed a } \\
\text { combination of an } \\
\text { ACEI or ARB and a } \\
\text { diuretic }\end{array}$} & Overall * & & 298 & 1464 & 20.4 & \\
\hline & \multirow[t]{2}{*}{ Rurality } & $\begin{array}{c}\text { Major cities of } \\
\text { Australia }\end{array}$ & 174 & 837 & 20.8 & 0.679 \\
\hline & & $\begin{array}{c}\text { Regional and Remote } \\
\text { Australia }\end{array}$ & 123 & 618 & 19.9 & \\
\hline & \multirow{2}{*}{ SEIFA quintile } & $\leq 3$ & 94 & 496 & 19.0 & 0.315 \\
\hline & & $>3$ & 203 & 958 & 21.2 & \\
\hline & \multirow{2}{*}{$\mathrm{CoC}$} & High & 104 & 528 & 19.7 & 0.639 \\
\hline & & Low & 194 & 936 & 20.7 & \\
\hline & \multirow{2}{*}{ CKD documented } & No & 148 & 751 & 19.7 & 0.527 \\
\hline & & Yes & 150 & 713 & 21.0 & \\
\hline & \multirow{2}{*}{ Systolic BP } & $>140 \mathrm{mmHg}$ & 143 & 643 & 22.2 & 0.123 \\
\hline & & $\leq 140 \mathrm{mmHg}$ & 150 & 792 & 19.0 & \\
\hline & \multirow{2}{*}{ Age } & $<65$ years & 74 & 444 & 16.7 & 0.021 \\
\hline & & $\geq 65$ years & 224 & 1020 & 22 & \\
\hline & \multirow{2}{*}{ Sex } & Female & 102 & 468 & 21.8 & 0.348 \\
\hline & & Male & 196 & 996 & 19.7 & \\
\hline
\end{tabular}


Table 4. Cont

\begin{tabular}{|c|c|c|c|c|c|c|}
\hline & Quality Indicator & & Numerator & Denominator & Percentage & $p$ Value \\
\hline \multirow{15}{*}{$\begin{array}{l}\text { 3. Percentage of } \\
\text { patients aged } 18 \text { to } 80 \\
\text { years with CKD } \\
\text { stages } 3-5, \\
\text { microalbuminuria } \\
\text { and diabetes treated } \\
\text { with multiple } \\
\text { antihypertensives } \\
\text { who are prescribed a } \\
\text { combination of an } \\
\text { ACEI or ARB and a } \\
\text { diuretic }\end{array}$} & Overall * & & 337 & 1634 & 20.6 & \\
\hline & \multirow{2}{*}{ Rurality } & $\begin{array}{l}\text { Major cities of } \\
\text { Australia }\end{array}$ & 190 & 956 & 19.9 & \multirow[t]{2}{*}{0.270} \\
\hline & & $\begin{array}{c}\text { Regional and Remote } \\
\text { Australia }\end{array}$ & 147 & 664 & 22.1 & \\
\hline & \multirow{2}{*}{ SEIFA quintile } & $\leq 3$ & 110 & 513 & 21.4 & \multirow[t]{2}{*}{0.672} \\
\hline & & $>3$ & 227 & 1106 & 20.5 & \\
\hline & \multirow{2}{*}{$\mathrm{CoC}$} & High & 144 & 641 & 22.5 & \multirow[t]{2}{*}{0.140} \\
\hline & & Low & 193 & 993 & 19.4 & \\
\hline & \multirow{2}{*}{ CKD documented } & No & 216 & 1075 & 20.1 & \multirow[t]{2}{*}{0.462} \\
\hline & & Yes & 121 & 556 & 21.8 & \\
\hline & \multirow{2}{*}{ Systolic BP } & $>140 \mathrm{mmHg}$ & 119 & 563 & 21.1 & \multirow[t]{2}{*}{0.667} \\
\hline & & $\leq 140 \mathrm{mmHg}$ & 213 & 1053 & 20.2 & \\
\hline & \multirow{2}{*}{ Age } & $<65$ years & 40 & 228 & 17.5 & \multirow[t]{2}{*}{0.215} \\
\hline & & $\geq 65$ years & 297 & 1406 & 21.1 & \\
\hline & \multirow{2}{*}{ Sex } & Female & 149 & 655 & 22.7 & \multirow[t]{2}{*}{0.083} \\
\hline & & Male & 188 & 979 & 19.2 & \\
\hline \multicolumn{7}{|l|}{$\begin{array}{l}\text { Treatment of } \\
\text { albuminuria }\end{array}$} \\
\hline \multirow{13}{*}{$\begin{array}{l}\text { 4. Percentage of } \\
\text { patients aged } 18 \text { to } \\
80 \text { years with CKD } \\
\text { stages } 3-5 \text { and } \\
\text { macroalbuminuria } \\
\text { who are prescribed an } \\
\text { ACEI or ARB }\end{array}$} & Overall * & & 1084 & 1741 & 62.3 & \\
\hline & Rurality & $\begin{array}{l}\text { Major cities of } \\
\text { Australia }\end{array}$ & 636 & 1016 & 62.6 & 0.725 \\
\hline & \multirow{3}{*}{ SEIFA quintile } & $\begin{array}{c}\text { Regional and Remote } \\
\text { Australia }\end{array}$ & 441 & 714 & 61.8 & \\
\hline & & $\leq 3$ & 353 & 573 & 61.6 & \multirow[t]{2}{*}{0.705} \\
\hline & & $>3$ & 723 & 1156 & 62.5 & \\
\hline & \multirow{2}{*}{$\mathrm{CoC}$} & High & 387 & 645 & 60.0 & \multirow[t]{2}{*}{0.135} \\
\hline & & Low & 697 & 1096 & 63.6 & \\
\hline & \multirow{2}{*}{ CKD documented } & No & 578 & 898 & 64.5 & \multirow[t]{2}{*}{0.046} \\
\hline & & Yes & 506 & 845 & 60.0 & \\
\hline & \multirow{2}{*}{ Age } & $<65$ years & 331 & 590 & 56.1 & \multirow[t]{2}{*}{$<0.001$} \\
\hline & & $\geq 65$ years & 753 & 1151 & 65.4 & \\
\hline & \multirow{2}{*}{ Sex } & Female & 327 & 544 & 60.1 & \multirow[t]{2}{*}{0.212} \\
\hline & & Male & 757 & 1197 & 63.2 & \\
\hline
\end{tabular}


Table 4. Cont

\begin{tabular}{|c|c|c|c|c|c|c|}
\hline & Quality Indicator & & Numerator & Denominator & Percentage & $p$ Value \\
\hline \multirow{13}{*}{$\begin{array}{l}\text { 5. Percentage of } \\
\text { patients aged } 18 \text { to } 80 \\
\text { years with CKD } \\
\text { stages } 3-5 \text {, } \\
\text { microalbuminuria } \\
\text { and diabetes who are } \\
\text { prescribed an ACEI or } \\
\text { ARB }\end{array}$} & Overall * & & 1252 & 1790 & 69.9 & \\
\hline & Rurality & $\begin{array}{l}\text { Major cities of } \\
\text { Australia }\end{array}$ & 738 & 1064 & 69.4 & 0.516 \\
\hline & \multirow{3}{*}{ SEIFA quintile } & $\begin{array}{l}\text { Regional and Remote } \\
\text { Australia }\end{array}$ & 502 & 709 & 70.8 & \\
\hline & & $\leq 3$ & 393 & 546 & 72.0 & 0.207 \\
\hline & & $>3$ & 846 & 1226 & 69.0 & \\
\hline & \multirow[b]{2}{*}{$\mathrm{CoC}$} & High & 502 & 705 & 71.2 & 0.348 \\
\hline & & Low & 750 & 1085 & 69.1 & \\
\hline & \multirow{2}{*}{ CKD documented } & No & 841 & 1179 & 71.3 & 0.075 \\
\hline & & Yes & 411 & 611 & 67.3 & \\
\hline & \multirow{2}{*}{ Age } & $<65$ years & 176 & 259 & 68 & 0.450 \\
\hline & & $\geq 65$ years & 1076 & 1531 & 70.3 & \\
\hline & \multirow{2}{*}{ Sex } & Female & 496 & 711 & 69.8 & 0.891 \\
\hline & & Male & 759 & 1079 & 70.1 & \\
\hline \multicolumn{7}{|l|}{ Prescription of statins } \\
\hline \multirow{11}{*}{$\begin{array}{l}\text { 6. Percentage of } \\
\text { patients aged } 50 \text { to } \\
65 \text { years with CKD } \\
\text { stages } 3-5 \text { who are } \\
\text { prescribed a statin }\end{array}$} & Overall * & & 1508 & 3693 & 40.8 & \\
\hline & Rurality & $\begin{array}{l}\text { Major cities of } \\
\text { Australia }\end{array}$ & 823 & 2023 & 40.7 & 0.898 \\
\hline & \multirow{3}{*}{ SEIFA quintile } & $\begin{array}{l}\text { Regional and Remote } \\
\text { Australia }\end{array}$ & 669 & 1636 & 40.9 & \\
\hline & & $\leq 3$ & 488 & 1077 & 45.3 & $<0.001$ \\
\hline & & $>3$ & 1004 & 2581 & 38.9 & \\
\hline & \multirow{2}{*}{$\mathrm{CoC}$} & High & 542 & 1292 & 42.0 & 0.311 \\
\hline & & Low & 966 & 2401 & 40.2 & \\
\hline & \multirow{2}{*}{ CKD documented } & No & 991 & 2547 & 38.9 & $<0.001$ \\
\hline & & Yes & 517 & 1146 & 45.1 & \\
\hline & \multirow{2}{*}{ Sex } & Female & 714 & 1814 & 39.4 & 0.073 \\
\hline & & Male & 794 & 1879 & 42.3 & \\
\hline \multicolumn{7}{|c|}{ Treatment of MBD } \\
\hline \multicolumn{3}{|c|}{$\begin{array}{l}\text { 7. Percentage of patients aged } 18 \text { to } 80 \text { years with CKD stages } 3-5 \text { and } \\
\text { with an elevated phosphate level who are prescribed a phosphate binder }\end{array}$} & 54 & 815 & 6.6 & \\
\hline \multicolumn{3}{|c|}{$\begin{array}{l}\text { 8. Percentage of patients aged } 18 \text { to } 80 \text { years with CKD stages } 3-5 \\
\text { treated with phosphate binders and with an elevated calcium level who } \\
\text { are prescribed a non-calcium-containing phosphate binder }\end{array}$} & 5 & 7 & 71.4 & \\
\hline
\end{tabular}


Table 4. Cont

\begin{tabular}{|c|c|c|c|c|c|c|}
\hline \multicolumn{3}{|c|}{ Quality Indicator } & Numerator & Denominator & Percentage & $p$ Value \\
\hline \multicolumn{3}{|c|}{$\begin{array}{l}\text { 9. Percentage of patients aged } 18 \text { to } 80 \text { years with CKD stages } 3-5 \\
\text { treated with phosphate binders and with a low calcium level who are } \\
\text { prescribed a calcium-containing phosphate binder }\end{array}$} & 6 & 12 & 50.0 & \\
\hline \multicolumn{7}{|c|}{ Medication safety } \\
\hline \multirow{13}{*}{$\begin{array}{l}\text { 10. Percentage of } \\
\text { patients } 18 \text { years or } \\
\text { older with CKD } \\
\text { stages } 3-5 \text { and a } \\
\text { prescription of RAS } \\
\text { blockers who are } \\
\text { prescribed at least } \\
\text { two RAS blockers } \\
\text { simultaneously }\end{array}$} & Overall* & & 1859 & 24,485 & 7.6 & \\
\hline & Rurality & $\begin{array}{c}\text { Major cities of } \\
\text { Australia }\end{array}$ & 1175 & 14,639 & 8.0 & 0.002 \\
\hline & \multirow{3}{*}{ SEIFA quintile } & $\begin{array}{l}\text { Regional and Remote } \\
\text { Australia }\end{array}$ & 678 & 9732 & 7.0 & \\
\hline & & $\leq 3$ & 587 & 7037 & 8.3 & 0.005 \\
\hline & & $>3$ & 1264 & 17,317 & 7.3 & \\
\hline & \multirow{2}{*}{$\mathrm{CoC}$} & High & 727 & 9756 & 7.5 & 0.499 \\
\hline & & Low & 1132 & 14,729 & 7.7 & \\
\hline & \multirow{2}{*}{ CKD documented } & No & 1339 & 18,073 & 7.4 & 0.069 \\
\hline & & Yes & 520 & 6412 & 8.1 & \\
\hline & \multirow{2}{*}{ Age } & $<65$ years & 182 & 2076 & 8.8 & 0.035 \\
\hline & & $\geq 65$ years & 1677 & 22,409 & 7.5 & \\
\hline & \multirow{2}{*}{ Sex } & Female & 1007 & 13,466 & 7.5 & 0.455 \\
\hline & & Male & 852 & 11,019 & 7.7 & \\
\hline \multicolumn{3}{|c|}{$\begin{array}{l}\text { 11. Percentage of patients } 18 \text { years or older with CKD stages } 3-5 \text { and } \\
\text { elevated calcium levels who are prescribed active vitamin D }\end{array}$} & 67 & 1343 & 5.0 & \\
\hline \multicolumn{3}{|c|}{$\begin{array}{l}\text { 12. Percentage of patients } 18 \text { years or older with CKD stages } 3-5 \text { and } \\
\mathrm{Hb} \geq 7.5 \text { who are prescribed ESA }\end{array}$} & 0 & 26,476 & 0.0 & \\
\hline \multirow{13}{*}{$\begin{array}{l}\text { 13. Percentage of } \\
\text { patients } 18 \text { years or } \\
\text { older with Egfr } \\
<30 \mathrm{~mL} / \mathrm{min} / 1.73 \mathrm{~m}^{2} \\
\text { who are prescribed a } \\
\text { NSAID }\end{array}$} & Overall * & & 735 & 5146 & 14.3 & \\
\hline & \multirow[t]{2}{*}{ Rurality } & $\begin{array}{l}\text { Major cities of } \\
\text { Australia }\end{array}$ & 421 & 3054 & 13.8 & 0.201 \\
\hline & & $\begin{array}{c}\text { Regional and Remote } \\
\text { Australia }\end{array}$ & 312 & 2072 & 15.1 & \\
\hline & \multirow{2}{*}{ SEIFA quintile } & $\leq 3$ & 238 & 1496 & 15.9 & 0.033 \\
\hline & & $>3$ & 494 & 3627 & 13.6 & \\
\hline & \multirow{2}{*}{$\mathrm{CoC}$} & High & 295 & 2058 & 14.3 & 0.935 \\
\hline & & Low & 440 & 3087 & 14.3 & \\
\hline & \multirow{2}{*}{ CKD documented } & No & 352 & 2367 & 14.9 & 0.266 \\
\hline & & Yes & 383 & 2779 & 13.8 & \\
\hline & \multirow{2}{*}{ Age } & $<65$ years & 68 & 724 & 9.4 & $<0.001$ \\
\hline & & $\geq 65$ years & 667 & 4422 & 15.1 & \\
\hline & \multirow{2}{*}{ Sex } & Female & 360 & 2648 & 13.6 & 0.147 \\
\hline & & Male & 375 & 2498 & 15.0 & \\
\hline
\end{tabular}


Table 4. Cont

\begin{tabular}{|c|c|c|c|c|c|c|}
\hline & Quality Indicator & & Numerator & Denominator & Percentage & $p$ Value \\
\hline \multirow{13}{*}{$\begin{array}{l}\text { 14. Percentage of } \\
\text { patients } 18 \text { years or } \\
\text { older with Egfr } \\
<30 \mathrm{~mL} / \mathrm{min} / 1.73 \mathrm{~m}^{2} \\
\text { and diabetes who are } \\
\text { prescribed metformin }\end{array}$} & \multicolumn{2}{|c|}{ Overall $^{*}$} & 278 & 1967 & 14.1 & \\
\hline & \multirow{2}{*}{ Rurality } & $\begin{array}{c}\text { Major cities of } \\
\text { Australia }\end{array}$ & 149 & 1208 & 12.3 & 0.005 \\
\hline & & $\begin{array}{c}\text { Regional and Remote } \\
\text { Australia }\end{array}$ & 126 & 749 & 16.8 & \\
\hline & \multirow{2}{*}{ SEIFA quintile } & $\leq 3$ & 80 & 608 & 13.2 & 0.444 \\
\hline & & $>3$ & 195 & 1349 & 14.4 & \\
\hline & \multirow{2}{*}{$\mathrm{CoC}$} & High & 119 & 829 & 14.4 & 0.810 \\
\hline & & Low & 159 & 1138 & 14.0 & \\
\hline & \multirow{2}{*}{ CKD documented } & No & 136 & 835 & 16.3 & 0.019 \\
\hline & & Yes & 142 & 1132 & 12.5 & \\
\hline & \multirow{2}{*}{ Age } & $<65$ years & 32 & 244 & 13.1 & 0.625 \\
\hline & & $\geq 65$ years & 246 & 1723 & 14.3 & \\
\hline & \multirow{2}{*}{ Sex } & Female & 127 & 957 & 13.3 & 0.285 \\
\hline & & Male & 151 & 1010 & 15 & \\
\hline \multirow{13}{*}{$\begin{array}{c}\text { 15. Percentage of } \\
\text { patients } 18 \text { years or } \\
\text { older with eGFR } \\
<50 \mathrm{~mL} / \mathrm{min} / 1.73 \mathrm{~m}^{2} \\
\text { who are prescribed } \\
\text { digoxin } \\
>0.125 \mathrm{mg} / \text { day }\end{array}$} & \multicolumn{2}{|c|}{ Overall * } & 995 & 26,434 & 3.8 & \\
\hline & \multirow[t]{2}{*}{ Rurality } & $\begin{array}{c}\text { Major cities of } \\
\text { Australia }\end{array}$ & 558 & 16,020 & 3.5 & 0.002 \\
\hline & & $\begin{array}{c}\text { Regional and Remote } \\
\text { Australia }\end{array}$ & 433 & 10,282 & 4.2 & \\
\hline & \multirow{2}{*}{ SEIFA quintile } & $\leq 3$ & 293 & 7394 & 4.0 & 0.295 \\
\hline & & $>3$ & 697 & 18,893 & 3.7 & \\
\hline & \multirow{2}{*}{$\mathrm{CoC}$} & High & 366 & 10,623 & 3.4 & 0.025 \\
\hline & & Low & 629 & 15,807 & 4.0 & \\
\hline & \multirow{2}{*}{ CKD documented } & No & 696 & 17,547 & 4.0 & 0.015 \\
\hline & & Yes & 299 & 8887 & 3.4 & \\
\hline & \multirow{2}{*}{ Age } & $<65$ years & 25 & 2252 & 1.1 & $<0.001$ \\
\hline & & $\geq 65$ years & 970 & 24,182 & 4.0 & \\
\hline & \multirow{2}{*}{ Sex } & Female & 596 & 14,411 & 4.1 & $<0.001$ \\
\hline & & Male & 399 & 12,023 & 3.3 & \\
\hline
\end{tabular}


Table 4. Cont

\begin{tabular}{|c|c|c|c|c|c|c|}
\hline \multicolumn{3}{|c|}{ Quality Indicator } & \multirow{2}{*}{$\begin{array}{c}\text { Numerator } \\
1160\end{array}$} & \multirow{2}{*}{$\begin{array}{c}\text { Denominator } \\
44,259\end{array}$} & \multirow{2}{*}{$\begin{array}{c}\text { Percentage } \\
2.6\end{array}$} & \multirow[t]{2}{*}{$p$ Value } \\
\hline \multirow{13}{*}{$\begin{array}{l}\text { 16. Percentage of } \\
\text { patients } 18 \text { years or } \\
\text { older with CKD } \\
\text { stages } 3-5 \text { and who } \\
\text { are prescribed with a } \\
\text { combination of } \\
\text { NSAID, RAS blocker } \\
\text { and diuretic }\end{array}$} & \multicolumn{2}{|c|}{ Overall* } & & & & \\
\hline & Rurality & $\begin{array}{c}\text { Major cities of } \\
\text { Australia }\end{array}$ & 663 & 26,617 & 2.5 & 0.032 \\
\hline & \multirow{3}{*}{ SEIFA quintile } & $\begin{array}{c}\text { Regional and Remote } \\
\text { Australia }\end{array}$ & 492 & 17,420 & 2.8 & \\
\hline & & $\leq 3$ & 397 & 12,254 & 3.2 & $<0.001$ \\
\hline & & $>3$ & 757 & 31,758 & 2.4 & \\
\hline & \multirow{2}{*}{$\mathrm{CoC}$} & High & 452 & 17,421 & 2.6 & 0.777 \\
\hline & & Low & 708 & 26,833 & 2.6 & \\
\hline & \multirow{2}{*}{ CKD documented } & No & 809 & 32,641 & 2.5 & 0.002 \\
\hline & & Yes & 351 & 11,618 & 3.0 & \\
\hline & \multirow{2}{*}{ Age } & $<65$ years & 86 & 4373 & 2.0 & 0.004 \\
\hline & & $\geq 65$ years & 1074 & 39,886 & 2.7 & \\
\hline & \multirow{2}{*}{ Sex } & Female & 640 & 24,165 & 2.6 & 0.691 \\
\hline & & Male & 520 & 20,094 & 2.6 & \\
\hline
\end{tabular}

$\mathrm{BP}$, blood pressure; CKD, chronic kidney disease; MBD, mineral and bone density; CoC, continuity of care; SEIFA, socio-economic indexes for areas; ACEI, angiotensin-converting enzyme inhibitor; ARB, angiotensin receptor blocker; RAS, renin-angiotensin system; eGFR, estimated glomerular filtration rate; ESAs, erythropoiesis-stimulating agents; NSAIDs, non-steroidal anti-inflammatory drugs. * 'Patient SEIFA', 'Patient Rurality', Patient $\mathrm{CoC}^{\prime}$, and 'CKD documented' for the indicator does not add up to 'Overall' due to missing data. 


\section{Discussion}

This study is the most extensive to date that evaluates the quality of medication prescribing in Australian general practice patients with CKD, utilising a set of 16 validated indicators and based on diabetes status [11]. Potential gaps in prescribing CKD progression protective medications and avoiding nephrotoxic drugs were identified. ACEIs/ARBs in patients with proteinuria or diabetes and statins in patients aged between 50 and 65 years were found to be under-prescribed. Potential inappropriate prescribing identified included simultaneous prescribing of at least two RAS inhibitors, prescribing of NSAIDs and metformin in patients with eGFR $<30 \mathrm{~mL} / \mathrm{min} / 1.73 \mathrm{~m}^{2}$, and use of high-dose digoxin in patients with an eGFR $<50 \mathrm{~mL} / \mathrm{min} / 1.73 \mathrm{~m}^{2}$. With at least one indicator, inappropriate prescribing was more common in patients with SEIFA $\leq 3$, aged $\geq 65$ years, or living in regional and remote Australia. Compared with patients without diabetes, patients with diabetes generally received more comprehensive blood pressure and laboratory monitoring and pharmacotherapy.

Despite strong evidence for the efficacy of ACEI/ARB to reduce proteinuria and slow progression of CKD to ESKD, less than 70\% of Australian adult patients with CKD stages 3-5 with diabetes and microalbuminuria were receiving an ACEI or ARB. The prescribing of an ACEI or ARB in patients with CKD with albuminuria was slightly lower in Australian general practice compared to that reported in other developed nations [24-27]. Studies from different provinces of Canada [24-26] investigating prescribing in CKD patients reported rates of $74 \%$ to $80 \%$ for ACEI or ARB prescribing, while a study conducted in the Netherlands found prescribing in $78 \%$ and $82 \%$ of non-diabetes and diabetes patients, respectively [11]. The reason for the low rate of ACEI/ARB prescribing could be non-concordance to Australian CKD treatment guidelines, including not referring patients to nephrology care [3]. The cost of ACEI/ARB probably had a limited impact on the rate of their prescription as these medications are subsidized by the Australian Pharmaceutical Benefits Scheme (PBS).

It was unexpected to find no difference or even low rates of an ACEI or ARB/an ACEI or ARB plus diuretic prescribing in patients with proteinuria and documented CKD compared to those without documented CKD. This might suggest that GPs awareness of patients' CKD status did not necessarily compel them to prescribe an ACEI/ARB. They may have other valid reasons for not prescribing, including hyperkalemia, hypotension, and acute renal injury (AKI) [28]. Among CKD patients with proteinuria who were receiving multiple antihypertensive agents, only a fifth use an ACEI/ARB in combination with a diuretic (double therapy) in this study. Double and triple (ACEI/ARB plus a diuretic plus an NSAID) therapies are associated with AKI [29], which might have discouraged GPs from prescribing. However, compared with those with triple therapy, the risk of developing AKI is less likely in patients with double therapy [29]. Combining ACEI/ARB with a thiazide diuretic instead of a loop diuretic might reduce the risk of discontinuation of ACEI/ARB [30]. The risk of inducing hypotension and the associated fall in elderly patients might outweigh the renoprotective effect gained by combining an ACEI/ARB with a diuretic, and it might have also prevented GPs from prescribing [30,31].

Statins are relatively well-tolerated medications and are beneficial in lowering the risk of cardiovascular events in patients with CKD [12,32]. Notwithstanding the PBS restrictions on the prescribing of statins, the current Kidney Disease: Improving Global Outcomes (KDIGO) and Kidney Health Australia's guidelines $[3,12]$ recommend statin or statin/ezetimibe treatment in adults aged 50 years and over with eGFR $<60 \mathrm{~mL} / \mathrm{min} / 1.73 \mathrm{~m}^{2}$ but not treated with chronic dialysis or kidney transplantation. In this study, only $40.8 \%$ of patients aged 50 to 65 years were receiving statins. These rates were less than a $54 \%$ lipid-lowering medication prescribing rate in primary care patients with CKD reported by a prior Australian study, AusHEART [33]. A study by Smits et al. [11] in the Netherlands using the same indicators reported a higher $(74 \%)$ rate of statin prescribing in patients with CKD stages $3-5$ aged 50 to 65 years. The significantly low rate of statin prescribing in those patients without documented CKD diagnosis (38.9\%) suggests that lack of CKD recognition by GPs might be one reason for the low rate of statin prescribing. 
Statin side effects and interactions were the main concerns of Australian patients taking statin [34], and which were also cited as the most commons reasons for statin discontinuation elsewhere [35]. The low rate of statin therapy in this study could be related to public concern over perceived adverse reactions following an extensive media campaign about the negative effect of statins [36]. The other likely reason is the lack of PBS subsidisation for statin therapy for CKD in the absence of other indications [37].

Our study indicated that patients living in relatively disadvantaged socio-economic areas (SEIFA score $\leq 3)$ were more likely to be prescribed potentially inappropriate NSAIDs, simultaneous use of at least two RAS inhibitors, and triple therapy (combined use of an NSAID, a RAS inhibitor and a diuretic). Similarly, patients from regional or remote areas of Australia were more likely to be prescribed potentially inappropriate digoxin, metformin, and triple therapy. These findings suggest that patients living in disadvantaged socio-economic areas, as well as regional and remote areas, may receive a lower quality of CKD care than patients living in socio-economic most advantaged areas or major cities. The health care inequality between regional/remote areas and major cities of Australia has been the subject of many reports and initiatives [38]. Three in five people in remote/very remote areas did not see a specialist because of distance, and people in outer regional and remote/very remote areas were less likely to have a usual GP [38]. Inequality in prescribing has also been found elsewhere. A study in France [39] reported that inappropriate prescribing was highest in older people living in municipalities with low socio-economic status. A similar study conducted in Ireland [40] also found that inappropriate prescribing was more prevalent in relatively deprived patients aged over 70 years.

In this study, patients with CKD and aged 65 years or over were more likely to be prescribed nephrotoxic medications: triple therapy, high-dose digoxin, and NSAIDs. One possible explanation is that some GPs may not consider an eGFR measurement between $45-59 \mathrm{~mL} / \mathrm{min} / 1.73 \mathrm{~m}^{2}$ as evidence of CKD in older individuals. They might consider these eGFR values as reflecting the normal physiological changes related to aging.

Unlike a previous study by Khanam et al. [31], using MedicineInsight data, which found higher $\mathrm{CoC}$ led to better blood pressure control, this study found no significant differences in prescribing quality between patients with higher and lower $\mathrm{CoC}$.

\section{Strengths and Limitations}

This study had a large sample size, and patient characteristics within the MedicineInsight dataset are similar to the Australian population $[2,14,16]$. However, there are several limitations. Medications prescribed solely by specialists, such as nephrologists and cardiologists, who worked in hospitals and speciality clinics were not recorded in NPS MedicineWise MedicineInsight. For instance, phosphate binders and ESAs are not usually prescribed by GPs (generally prescribed by nephrologists), and thus our data were not complete on the use of these medications.

We did not account for medication contraindications and adverse drug reactions that may have prevented GPs from prescribing a specific class of medication to patients. Adverse drug reactions are recorded in free text in 'Allergies/Reactions Table' in the NPS MedicineWise MedicineInsight dataset. This table is not an event-based table and does not necessarily record each occurrence of adverse drug reaction. Free-text search for an adverse drug reaction from this table is of poor quality.

NSAIDs are also available without a prescription, but we could only obtain data on prescribed NSAIDs. Simultaneous prescribing of at least two RAS blockers within the four months might not necessarily indicate concomitant inappropriate use. It might be an overlapping period of switching from one RAS blocker monotherapy to the other. We also did not investigate the impact of medication use on patient outcomes.

GPs collected the data for clinical decision making, not for research purposes. The EHRs may not contain all sociodemographic and clinical characteristics. For instance, indigenous status was not recorded for $24.3 \%$ of the patients. There is a possibility that aspects of patients' medical history, 
prescriptions, and laboratory tests were recorded in notes and not included in the research data, which used specified fields and not the body of free-text consultation notes.

We noted that including only regular patients (who had three or more clinical encounters in past two years) in this study potentially introduced selection bias by including more older patients with multiple comorbidities who visited their GP more frequently. However, four in five Australian patients visited their GP multiple times in a year [41], and nearly all patients could visit their GP at least three times in two years. In conclusion, we identified the potential for possible improvement in the prescribing of recommended preventive medications and deprescribing of nephrotoxic medication in patients with CKD in Australian primary care. Programmes to optimise the quality use of medications should focus on improving the prescribing practices for protective medications, such as an ACEI or ARB and a statin, and deprescribing concurrent NSAIDs and RAS blockers in patients with CKD.

Supplementary Materials: The data we used for this study is stored only in Australia and can be obtained from MedicineInsight. The following are available online at http://www.mdpi.com/2077-0383/9/3/783/s1, Figure S1: Appropriate prescribing in patients with chronic kidney disease (CKD) stages 3-5 assessed with prescribing quality indicators (PQIs), Figure S2: Potential inappropriate prescribing assessed with five prescribing quality indicators (PQIs).

Author Contributions: W.M.B., A.K., G.M.P., L.R.B., M.J. and J.R. conceived the study design. A.K. and T.S. analysed the data. W.M.B. wrote the first draft of the manuscript. All authors (W.M.B., A.K., T.S., J.R., L.R.B., G.M.P., M.J. and B.C.W.) reviewed and provided feedback several times on the manuscript. All authors have read and agreed to the published version of the manuscript.

Funding: This research received no external funding.

Acknowledgments: The authors would like to acknowledge the NPS MedicineWise MedicineInsight for providing the data.

Conflicts of Interest: The authors declare no conflict of interest.

Consent for Publication: Not applicable.

\section{Abbreviations}

$\begin{array}{ll}\text { ACEIs } & \text { angiotensin-converting enzyme inhibitors } \\ \text { ACR } & \text { albumin-to-creatinine ratio } \\ \text { AKI } & \text { acute kidney injury } \\ \text { ARBs } & \text { angiotensin receptor blockers } \\ \text { ATC } & \text { anatomical therapeutic chemical } \\ \text { CKD } & \text { chronic kidney disease } \\ \text { CoC } & \text { continuity of care } \\ \text { EHRs } & \text { electronic health records } \\ \text { eGFR } & \text { estimated glomerular filtration rate } \\ \text { ESAs } & \text { erythropoiesis-stimulating agents } \\ \text { GPs } & \text { general practitioners } \\ \text { KDIGO } & \text { Kidney Disease: Improving Global Outcomes } \\ \text { NSAIDs } & \text { non-steroidal anti-inflammatory drugs } \\ \text { PQIs } & \text { prescribing quality indicators } \\ \text { RAS } & \text { renin-angiotensin system } \\ \text { SEIFA } & \text { socio-economic indexes for areas }\end{array}$

\section{References}

1. Australian Bureau of Statistics. National Health Survey: First Results, 2014-15. Kidney Disease; Australian Bureau of Statistics: Canberra, Australia, 2015.

2. Radford, J.; Kitsos, A.; Stankovich, J.; Castelino, R.; Khanam, M.; Jose, M.; Peterson, G.; Saunder, T.; Wimmer, B.; Razizaidi, T. The epidemiology of chronic kidney disease in Australian general practice: National prescribing service medicinewise medicineinsight dataset. Nephrology 2018, 24, 1017-1025. [CrossRef] [PubMed]

3. Kidney Health Australia. Chronic Kidney Disease (Ckd) Management in General Practice, 3rd ed.; The Australian Kidney Foundation: Melbourne, Australia, 2015.

4. Australian Institute of Health and Welfare 2019. Chronic Kidney Disease; AIHW: Canberra, Australia, 2019. 
5. Mann, J.F.; Schmieder, R.E.; McQueen, M.; Dyal, L.; Schumacher, H.; Pogue, J.; Wang, X.; Maggioni, A.; Budaj, A.; Chaithiraphan, S.; et al. Renal outcomes with telmisartan, ramipril, or both, in people at high vascular risk (the ontarget study): A multicentre, randomised, double-blind, controlled trial. Lancet 2008, 372, 547-553. [CrossRef]

6. Whittaker, C.F.; Miklich, M.A.; Patel, R.S.; Fink, J.C. Medication safety principles and practice in Ckd. Clin. J. Am. Soc. Nephrol. 2018, 13, 1738-1746. [CrossRef] [PubMed]

7. Venuthurupalli, S.K.; Healy, H.; Fassett, R.; Cameron, A.; Wang, Z.; Hoy, W.E. Chronic kidney disease, Queensland: Profile of patients with chronic kidney disease from regional Queensland, Australia: A registry report. Nephrology 2019, 24, 1257-1264. [CrossRef]

8. Manski-Nankervis, J.E.; Thuraisingam, S.; Sluggett, J.K.; Lau, P.; Blackberry, I.; Ilomaki, J.; Furler, J.; Bell, J.S. Prescribing for people with type 2 diabetes and renal impairment in Australian general practice: A national cross sectional study. Prim. Care Diabetes 2019, 13, 113-121. [CrossRef]

9. Hui, K.; Nalder, M.; Buising, K.; Pefanis, A.; Ooi, K.Y.; Pedagogos, E.; Nelson, C.; Kirkpatrick, C.M.J.; Kong, D.C.M. Patterns of use and appropriateness of antibiotics prescribed to patients receiving haemodialysis: An observational study. BMC Nephrol. 2017, 18, 156. [CrossRef]

10. Doody, H.K.; Peterson, G.M.; Watson, D.; Castelino, R.L. Retrospective evaluation of potentially inappropriate prescribing in hospitalized patients with renal impairment. Curr. Med. Res. Opin. 2015, 31, 525-535. [CrossRef]

11. Smits, K.P.; Sidorenkov, G.; Bilo, H.J.; Bouma, M.; Van Ittersum, F.J.; Voorham, J.; Navis, G.; Denig, P. Development and initial validation of prescribing quality indicators for patients with chronic kidney disease. Nephrol. Dial. Transplant. 2016, 31, 1876-1886. [CrossRef]

12. Wanner, C.; Tonelli, M. Kdigo clinical practice guideline for lipid management in Ckd: Summary of recommendation statements and clinical approach to the patient. Kidney Int. 2014, 85, 1303-1309. [CrossRef]

13. Wheeler, D.C.; Becker, G.J. Summary of KDIGO guideline. What do we really know about management of blood pressure in patients with chronic kidney disease? Kidney Int. 2013, 83, 377-383. [CrossRef]

14. Gonzalez-Chica, D.A.; Vanlint, S.; Hoon, E.; Stocks, N. Epidemiology of arthritis, chronic back pain, gout, osteoporosis, spondyloarthropathies and rheumatoid arthritis among 1.5 million patients in Australian general practice: Nps medicinewise medicineinsight dataset. BMC Musculoskelet. Disord. 2018, $19,20$. [CrossRef] [PubMed]

15. Gadzhanova, S.; Pratt, N.; Roughead, E. Use of SGLT2 inhibitors for diabetes and risk of infection: Analysis using general practice records from the NPS medicinewise medicineinsight program. Diabetes Res. Clin. Pract. 2017, 130, 180-185. [CrossRef] [PubMed]

16. MedicineInsight. Medicineinsight Data Book and Data Dictionary; NPS MedicineWise: Sydney, Australia, 2018.

17. Johnson, D.W.; Jones, G.R.; Mathew, T.H.; Ludlow, M.J.; Doogue, M.P.; Jose, M.D.; Langham, R.G.; Lawton, P.D.; McTaggart, S.J.; Peake, M.J.; et al. Chronic kidney disease and automatic reporting of estimated glomerular filtration rate: New developments and revised recommendations. Med. J. Aust. 2012, 197, 224-225. [CrossRef] [PubMed]

18. Australian Bureau of Statistics. Socio-Economic Indexes for Areas (Seifa); Australian Bureau of Statistics: Belconnen, Australia, 2018.

19. Australian Statistical Geography Standard (ASGS). Significant Urban Areas, Urban Centres and Localities, Section of State, July 2016; Australian Bureau of Statistics: Belconnen, Australia, 2017.

20. Pollack, C.E.; Hussey, P.S.; Rudin, R.S.; Fox, D.S.; Lai, J.; Schneider, E.C. Measuring care continuity: A comparison of claims-based methods. Med. Care 2016, 54, e30-e34. [CrossRef] [PubMed]

21. Maarsingh, O.R.; Henry, Y.; Van de Ven, P.M.; Deeg, D.J.H. Continuity of care in primary care and association with survival in older people: A 17-year prospective cohort study. Br. J. Gen. Pract. 2016, 66, e531-e539. [CrossRef] [PubMed]

22. Pereira Gray, D.J.; Sidaway-Lee, K.; White, E.; Thorne, A.; Evans, P.H. Continuity of care with doctors-A matter of life and death? A systematic review of continuity of care and mortality. BMJ Open 2018, 8, e021161. [CrossRef]

23. R Core Team. R: A Language and Environment for Statistical Computing; R Foundation for Statistical Computing: Vienna, Austria, 2018. 
24. Nash, D.M.; Brimble, S.; Markle-Reid, M.; McArthur, E.; Tu, K.; Nesrallah, G.E.; Grill, A.; Garg, A.X. Quality of care for patients with chronic kidney disease in the primary care setting: A retrospective cohort study from Ontario, Canada. Can. J. Kidney Health Dis. 2017, 4. [CrossRef]

25. Tu, K.; Bevan, L.; Hunter, K.; Rogers, J.; Young, J.; Nesrallah, G. Quality indicators for the detection and management of chronic kidney disease in primary care in Canada derived from a modified Delphi panel approach. CMAJ Open 2017, 5, E74-E81. [CrossRef]

26. Manns, L.; Scott-Douglas, N.; Tonelli, M.; Weaver, R.; Tam-Tham, H.; Chong, C.; Hemmelgarn, B. A population-based analysis of quality indicators in Ckd. Clin. J. Am. Soc. Nephrol. 2017, 12, 727-733. [CrossRef]

27. Jameson, K.; Jick, S.; Hagberg, K.W.; Ambegaonkar, B.; Giles, A.; O’Donoghue, D. Prevalence and management of chronic kidney disease in primary care patients in the UK. Int. J. Clin. Pract. 2014, 68, 1110-1121. [CrossRef]

28. Ahmed, A.; Jorna, T.; Bhandari, S. Should we stop angiotensin converting enzyme inhibitors/angiotensin receptor blockers in advanced kidney disease? Nephron 2016, 133, 147-158. [CrossRef] [PubMed]

29. Lapi, F.; Azoulay, L.; Yin, H.; Nessim, S.J.; Suissa, S. Concurrent use of diuretics, angiotensin converting enzyme inhibitors, and angiotensin receptor blockers with non-steroidal anti-inflammatory drugs and risk of acute kidney injury: Nested case-control study. BMJ 2013, 346, e8525. [CrossRef] [PubMed]

30. Qiao, Y.; Shin, J.I.; Sang, Y.; Inker, L.A.; Secora, A.; Luo, S.; Coresh, J.; Alexander, G.C.; Jackson, J.W.; Chang, A.R.; et al. Discontinuation of angiotensin converting enzyme inhibitors and angiotensin receptor blockers in chronic kidney disease. Mayo Clin. Proc. 2019, 94, 2220-2229. [CrossRef] [PubMed]

31. Khanam, M.A.; Kitsos, A.; Stankovich, J.; Castelino, R.; Jose, M.; Peterson, G.M.; Wimmer, B.; Zaidi, T.R.; Radford, J. Association of continuity of care with blood pressure control in patients with chronic kidney disease and hypertension. Aust. J. Gen. Pract. 2019, 48, 300-306. [CrossRef] [PubMed]

32. Vassalotti, J.A.; Centor, R.; Turner, B.J.; Greer, R.C.; Choi, M.; Sequist, T.D. Practical approach to detection and management of chronic kidney disease for the primary care clinician. Am. J. Med. 2016, 129, $153-162$. [CrossRef] [PubMed]

33. Razavian, M.; Heeley, E.L.; Perkovic, V.; Zoungas, S.; Weekes, A.; Patel, A.A.; Anderson, C.S.; Chalmers, J.P.; Cass, A. Cardiovascular Risk management in chronic kidney disease in general practice (the ausheart study). Nephrol. Dial. Transplant. 2012, 27, 1396-1402. [CrossRef] [PubMed]

34. Deckx, L.; Kreijkamp-Kaspers, S.; McGuire, T.; Bedford, S.; Van Driel, M. Chewing the fat over statins: Consumer concerns about lipid-lowering medication. Aust. Fam. Physician 2017, 46, 594-601.

35. Jacobson, T.A.; Cheeley, M.K.; Jones, P.H.; La Forge, R.; Maki, K.C.; Lopez, J.A.G.; Xiang, P.; Bushnell, D.M.; Martin, M.L.; Cohen, J.D. The statin adverse treatment experience survey: Experience of patients reporting side effects of statin therapy. J. Clin. Lipidol. 2019, 13, 415-424. [CrossRef]

36. Nordestgaard, B.G. Why do the media report negative news about statins? Eur. Heart J. 2018, 39, 337-338. [CrossRef]

37. Kennard, A.; Singer, R. Lipid lowering in renal disease. Aust. Prescr. 2017, 40, 141-146. [CrossRef]

38. Australian Institute of Health Welfare. Survey of Health Care: Selected Findings for Rural and Remote Australians; AIHW: Canberra, Australia, 2018.

39. Beuscart, J.B.; Genin, M.; Dupont, C.; Verloop, D.; Duhamel, A.; Defebvre, M.M.; Puisieux, F. Potentially inappropriate medication prescribing is associated with socioeconomic factors: A spatial analysis in the French Nord-Pas-de-Calais region. Age Ageing 2017, 46, 607-613. [CrossRef] [PubMed]

40. Odubanjo, E.; Bennett, K.; Feely, J. Influence of socioeconomic status on the quality of prescribing in the elderly: A population based study. Br. J. Clin. Pharmacol. 2004, 58, 496-502. [CrossRef] [PubMed]

41. The Royal Australian College of General Practitioners. General Practice: Health of the Nation 2019; The Royal Australian College of General Practitioners: East Melbourne, Australia, 2019.

(C) 2020 by the authors. Licensee MDPI, Basel, Switzerland. This article is an open access article distributed under the terms and conditions of the Creative Commons Attribution (CC BY) license (http://creativecommons.org/licenses/by/4.0/). 